\title{
The time-dependent effects of bipolar radiofrequency energy on bovine articular cartilage
}

\author{
Liangquan Peng ${ }^{1,2,4,5,6+}$, Yusheng Li ${ }^{3+}$, Kai Zhang ${ }^{1}$, Qi Chen', Lulu Xiao', Yiyun Geng ${ }^{2}$, Yong Huang ${ }^{2,4}$, \\ Weimin Zhu' ${ }^{1,2}$, Wei Lu', Greg Zhang ${ }^{7}$, Zhenhan Deng ${ }^{1,2,4,5,6^{*}}$ and Daping Wang ${ }^{1,2,4,5,6^{*}}$
}

\begin{abstract}
Purpose: The purpose of this study was to compare the effect of bipolar radiofrequency energy (bRFE) on chondroplasty at the different time durations in an in vitro experiment that simulated an arthroscopic procedure.

Methods: Six fresh bovine knees were used in our study. Six squares were marked on both the medical and lateral femoral condyles of each femur. Each square was respectively treated with bRFE for $0 \mathrm{~s}, 10 \mathrm{~s}, 20 \mathrm{~s}, 30 \mathrm{~s}, 40 \mathrm{~s}$ and 50 s. Full-thickness articular cartilage specimens were harvested from the treatment areas. Each specimen was divided into three distinct parts: one for hematoxylin/eosin staining histology, another for cartilage surface contouring assessment via scanning electron microscopy (SEM), and the last one for glycosaminoglycan (GAG) content measurement.

Results: bRFE caused time-correlated damage to chondrocytes, and GAG content in the cartilage was negatively correlated to exposure time. bRFE caused time-correlated damage to chondrocytes. The GAG content in the cartilage negatively correlated with the exposure time. The sealing effect positively correlated with the exposure time. Additionally, it took at least $20 \mathrm{~s}$ of radiofrequency exposure to render a smooth cartilage surface and a score of 2 (normal) in the scoring system used.

Conclusion: bRFE usage in chondroplasty could effectively trim and polish the cartilage lesion area; however, it induces a dose-dependent detrimental effect on chondrocytes and metabolic activity that negatively correlated with the treatment time. Therefore, cautions should be taken in the use of bRFE for treatment of articular cartilage injury.
\end{abstract}

Keywords: Radiofrequency energy, Chondroplasty, Articular cartilage, Chondrocyte, Scanning electron microscope

\section{Introduction}

Osteoarthritis results from the degeneration of articular cartilage and the ensuing secondary hyperostosis. Partial-thickness chondral defect has been found responsible for the degeneration of articular cartilage and

\footnotetext{
* Correspondence: dengzhenhan@email.szu.edu.cn; dapingwang1963@qq.com

'Liangquan Peng and Yusheng Li contributed equally to this work. 'Department of Sports Medicine, the First Affiliated Hospital of Shenzhen University, Shenzhen Second People's Hospital, Shenzhen 518035, Guangdong, China

Full list of author information is available at the end of the article
}

remains without effective treatment methods $[1,2]$. In recent years, bipolar radiofrequency energy (bRFE) ablation has gained popularity among arthroscopic knee surgeries as a solution to articular cartilage lesions; it creates a smooth, stable cartilage surface by thermal shrinkage or removal of fibrillated cartilage and thus stops the progression of chondral degeneration [3].

After studying chondromalacic human cartilage, Edwards et al. [4] reported that radiofrequency caused cellular death at $2 \mathrm{~mm}$ depth, and the damage even reached subchondral bone among 13 out of 20

(c) The Author(s). 2020 Open Access This article is licensed under a Creative Commons Attribution 4.0 International License, which permits use, sharing, adaptation, distribution and reproduction in any medium or format, as long as you give appropriate credit to the original author(s) and the source, provide a link to the Creative Commons licence, and indicate if changes were made. The images or other third party material in this article are included in the article's Creative Commons licence, unless indicated otherwise in a credit line to the material. If material is not included in the article's Creative Commons licence and your intended use is not permitted by statutory regulation or exceeds the permitted use, you will need to obtain permission directly from the copyright holder. To view a copy of this licence, visit http://creativecommons.org/licenses/by/4.0/. The Creative Commons Public Domain Dedication waiver (http://creativecommons.org/publicdomain/zero/1.0/) applies to the data made available in this article, unless otherwise stated in a credit line to the data. 
specimens. In an in vitro arthroscopic experiment, Ryan et al. [5] highlighted that bRFE hindered cartilage vitality and damaged its matrix when the power was above 20 W. Therefore, when bRFE is applied to chondral conditions, the power should be controlled within $20 \mathrm{~W}$, and the safety profile of radiofrequency remains yet to be proved in long-term studies. Voloshin et al. [6] demonstrated chondroplasty with bRFE was indeed effective for partial-thickness chondral defects, but it is still awaiting long-term results. A recent systematic review showed that bRFE ablation achieves dramatically better clinical outcomes and lower complication rates in the treatment of chondral defects than a mechanical shaving device [7]. However, thermal chondroplasty using bRFE ablation also arouses concerns about the risk of osteonecrosis, chondrolysis, and progression of partial-thickness chondral lesions [8].

In summation, the safety and long-term effects of bRFE, especially in regards to chondral lesions, are major concerns of practitioners and patients [9]. Therefore, the current study aimed to investigate how the different exposure times of bRFE affect the vitality of chondrocytes and cartilage surface. The study simulated the clinical treatment of arthroscopic procedure in an in vitro setup and applied a constant level of power to the radiofrequency. Our study will contribute to current literature on the clinical use of radiofrequency in resolving chondral defects.

\section{Materials and methods}

\section{Subjects}

The animal experiment was carried out in accordance with relevant guidelines and regulations and was approved by the Medical Ethics Committee of the First Affiliated Hospital of Shenzhen University. Fresh adult bovine knees purchased from the Guangdong Medical Laboratory Animal Center were used in this experiment. The animals were around 18 months old with closed epiphyses. The entire knee joint was harvested with the joint capsule intact and stored at $4{ }^{\circ} \mathrm{C}$ and delivered to the lab within 1 day. Chondrocyte viability was maintained during a 3- to 6-day period of storage, which has been demonstrated by a previous publication [10]. The experiment was performed 1 day after the animals' death.

\section{Experimental model}

Under sterile conditions, a model of grade-II cartilage degeneration was established by opening the knee joints, exposing the articular cartilage on the femoral condyles, scraping chondral membrane off with scalpel, and abrading the cartilage on both the medial and lateral condyles with a rasp [5]. Grids consisting of six squares of $1 \times 1$ $\mathrm{cm}^{2}$ area were created on the medial and lateral femoral condyles at the weight-bearing area with a sterile ink marker. Each grid was marked with a number from 0 to 5 (Fig. 1a). Zero represented the control without any bRFE treatment, while the others served as the experiment groups treated with different time durations of bRFE ( $N=6$ /group).

\section{Treatment with bRFE}

Radiofrequency ablation was generated from the SAPHYRE $60^{\circ}$ angle bipolar ablation probe on the Vulcan EAS electrothermal system (Smith and Nephew, Inc., Andover, MA, USA). The probe was set at bipolar ablation mode at $70 \mathrm{~W}$. The probe was moved in a meandering pattern in contact with the cartilage surface but not applying and pressure; in addition, no fluid flow was used during bRFE treatment. The model was submerged in normal saline in order to simulate the arthroscopic environment. Squares no. 1 to 5 were treated in the aforementioned method for $10,20,30,40$, and $50 \mathrm{~s}$, respectively. After the treatment, the squares were taken off one by one with a scalpel blade ensuring the removal of full-thickness cartilage containing subchondral bone. Each cartilage square was dissected into three parts: part A, B, and C. Part A was used for hematoxylin/eosin (HE) staining. Part B was used for observation of the cartilage contour under scanning electron microscope (SEM). Part $\mathrm{C}$ was used for the

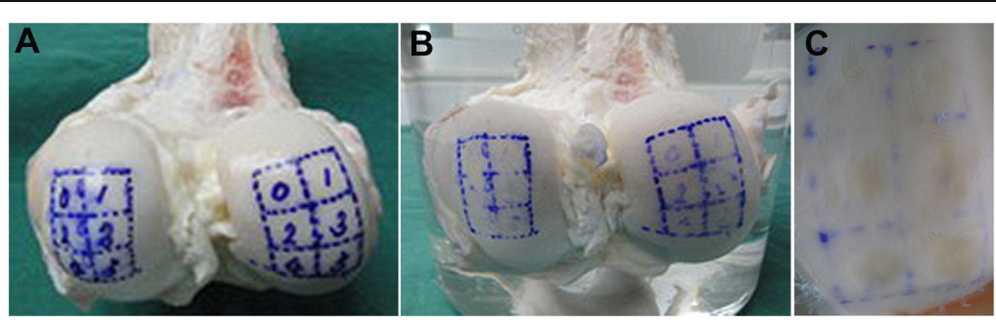

Fig. 1 a Groups at the medial and lateral distal bovine femoral condyles. The 0-s group, treated with bRFE for 0 s; the 10-s group, treated with bRFE for 10s; the 20-s group, treated with bRFE for 20 s; the 30-s group, treated with bRFE for $30 \mathrm{~s}$; the 40-s group, treated with bRFE for 40 s; the 50-s group, treated with bRFE for $50 \mathrm{~s}$. b Medial and lateral distal bovine femoral condyles before RFE treatment. c Medial and lateral distal bovine femoral condyles after RFE treatment 
measurement of glycosaminoglycan (GAG) content in the articular cartilage.

\section{Histology}

All the part A's from the six groups were fixed in 10\% neutral buffered formalin (NBF) for 2 days. Then, they were decalcified and paraffin-embedded. Sections were cut at $5-\mu \mathrm{m}$ thickness using a microtome, deparaffinized through xylene, and hydrated via ethanol gradient and water. Afterwards, HE staining was performed to reveal the cartilage morphology. The slices were dyed in hematoxylin solution for $5 \mathrm{~min}$, given a 1 -min water soak, differentiated with $1 \%$ hydrochloric acid ethanol for $30 \mathrm{~s}$, given a 15 -min water soak, dyed with $0.5 \%$ eosin for $3 \mathrm{~min}$, given a distilled water soak, and finally sealed for observation after dehydration.

\section{SEM observation}

Part B was fixed in 10\% NBF, dehydrated in a graded series of ethanol, dried at critical point, and coated with gold in an Autoconductavac IV (Seevac, Pittsburgh, PA) before their contouring was examined with SEM (Hitachi S $3000 \mathrm{~N}$, Tokyo, Japan) and scored according to the system provided in Table 1 [11]. A higher score indicates a smoother articular cartilage surface.

\section{GAG content}

GAG content was measured using the described dimethylmethylene blue (DMB) method [12]. After freeze-drying for 1 day, the cartilage specimens (part C) were weighed on an electronic scale to determine their dry weight. Then, they were immersed in papain at $60^{\circ} \mathrm{C}$ 1 day for enzymolysis. Three milliliters of DMB was added for every $100 \mu \mathrm{l}$ of the solution. The optical density (OD) value was determined using a UV spectrophotometer. Compared with standard curves, the GAG content of each specimen was calculated using the following formula: GAG content $(\mu \mathrm{g} / \mathrm{mg})=$ GAG content of the specimen/dry weight of the specimen.

\section{Statistical analysis}

Sample results are presented in the text as mean \pm standard deviation. The software SPSS 16.0 (version 15.0 for Windows; SPSS Inc., Chicago, IL, USA) was

Table 1 SEM grading system for chondromalacic cartilage surface after bRFE treatment

\begin{tabular}{ll}
\hline Surface of chondromalacic cartilage & Score \\
\hline Extremely smooth & 3 \\
Relatively smooth with melted fronds & 2 \\
Rough and irregular with melted fronds & 1 \\
Rough and irregular with fronds & 0
\end{tabular}

If a surface was graded to be between 2 scores, the mean of the 2 scores was used $(0.5,1.5,2.5)$ applied for statistical analysis and management. The one-way analysis of variance (ANOVA), SNK-q, and Dunnett's T3 were applied for comparisons of multisample means and heterogeneity of variance. Results were considered significant at a value of $P<0.05$.

\section{Results}

\section{Macroscopic observation}

The gross appearance of the articular cartilage before and after rRFE treatment was presented in Fig. 1b, c. The control group showed a rough and uneven cartilage surface with a bright white color. In the 10-s and 20 -s treatment groups, the cartilage fragment was dissolved, and the surface was slightly rough without any change in color. In the 30-s and 40-s treatment groups, the surface was smoother with a light yellow color. In the 50-s treatment group, the surface was smooth and became yellow in color.

\section{Histology}

We observed cracks on the rough surface and chondrocytes with normal morphology orderly arranged in each layer in the control group. The cartilage surface became smoother, and the chondrocytes appeared more vacuolated, had a disorderly arrangement, and decreased in number along with the increasing bRFE treatment time in the experiment groups (Fig. 2).

\section{SEM examination}

The results of SEM examination indicated a positive correlation between smoothness of cartilage surface and the exposure time to bipolar frequency (Fig. 3). The cartilage surface did not become smooth and the score did not reach 2 (normal) until $20 \mathrm{~s}$ of frequency exposure. The SEM score was remarkably higher among 20-s, 30-s, 40$\mathrm{s}$, and 50-s treatment groups than the 10-s treatment and control groups. Comparison of SEM scores among groups revealed that a statistically significant difference was present in all the results except for that between the 30 -s and 40-s groups as well as that between the 40-s and 50-s groups (Fig. 4).

\section{GAG content}

The GAG content in the cartilage specimens was negatively correlated with the bRFE exposure time. All of the pair-wise comparisons between groups were statistically significant except for that between the 40-s and 50-s treatment groups (Fig. 5).

\section{Discussion}

Chondroplasty is commonly applied to chondral lesions in clinical practice, although there are multiple alternatives. Laser is the first approach to chondroplasty with valid efficacy, but was later rendered 


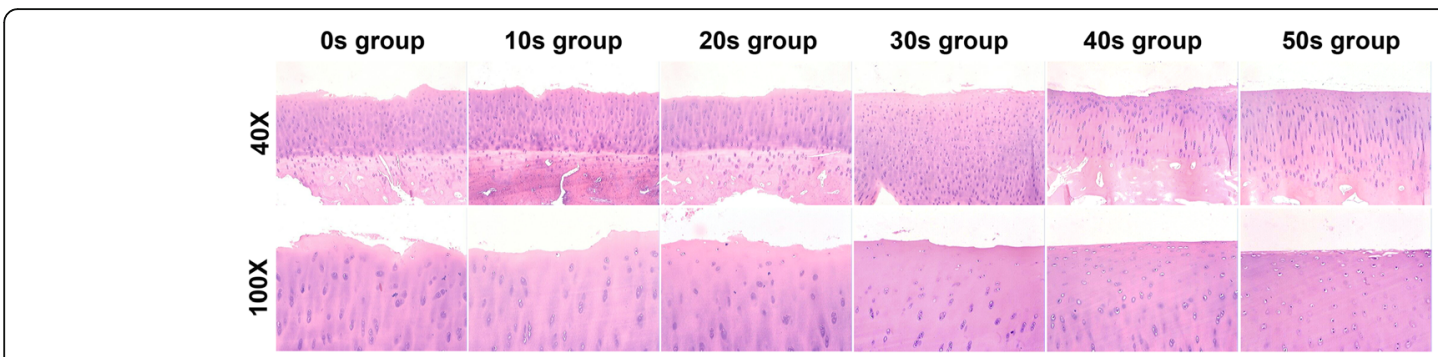

Fig. 2 HE staining showed the cartilage morphology in each group after different duration times of bRFE treatment

obsolete due to iatrogenic subchondral osteonecrosis and its high cost $[13,14]$. On the other hand, mechanical debridement (with shaver) intends to eliminate fibrillated or loose cartilage; however, it also removes healthy surrounding articular cartilage and does not improve the clefts and instability on the cartilaginous surface $[15,16]$.

Recently, thermal chondroplasty with radiofrequency has gained a wider acceptance. Monopolar and bipolar radiofrequency devices still prevail in clinical practice. The outcome of radiofrequency is affected by multiple variables, including treatment protocol, power setting, exposure time, and the temperature and speed of fluid flow. As safety and efficacy are the prerequisites for radiofrequency in chondroplasty [17], our study reproduced the actual surgical model to facilitate the observation of the impact of exposure time of bRFE on chondrocyte vitality and surface contouring under simulated arthroscopic environment and constant power delivery. These two major outcome measures enabled us to evaluate the safety and efficacy of this treatment method.

A previous study had demonstrated that the heat produced by bRFE could damage both chondrocytes and matrix [18]. GAG, produced and released by chondrocytes and a major ingredient of chondroid matrix, fills in the network built by type-II collagen. Kaplan et al. [19] revealed that radiofrequency sealed the cartilaginous surface while sparing the surrounding chondrocyte and matrix. Viable cell staining followed by confocal laser microscopy has been commonly used in research into bRFE-induced chondrocytic death, and signs of compromised vitality and metabolic activity of chondrocytes adjacent to the treatment zone have been noticed [10]. Hence, the GAG content could better reflect the physiological health of chondrocytes. This perspective is underpinned by the fact that proteoglycan has a significantly higher metabolic rate than collagen, making it feasible to use on an in vitro assessment of chondrocyte vitality $[5,18]$. It has been demonstrated that the volume of GAG released from cartilage dropped as exposure time increased under constant energy output, indicating that prolonged exposure results in lower cartilage metabolic activity.

Cartilage smoothness may be overestimated under arthroscopic exploration due to its lower magnification power than SEM, leading to insufficient sealing of the clefts at the end of the procedure [11]. In our study, the score of 2, indicating a relatively smooth contour according to the SEM grading system, was only possible when the cartilage was exposed to bRFE for longer than $20 \mathrm{~s}$. The cartilage smoothness being correlated to the exposure time of bRFE is consistent with that of Lu et al. [11].

Bovine knee was selected as the specimen based on the similarity in thickness to human articular cartilage as well as shared biomechanical and biochemical

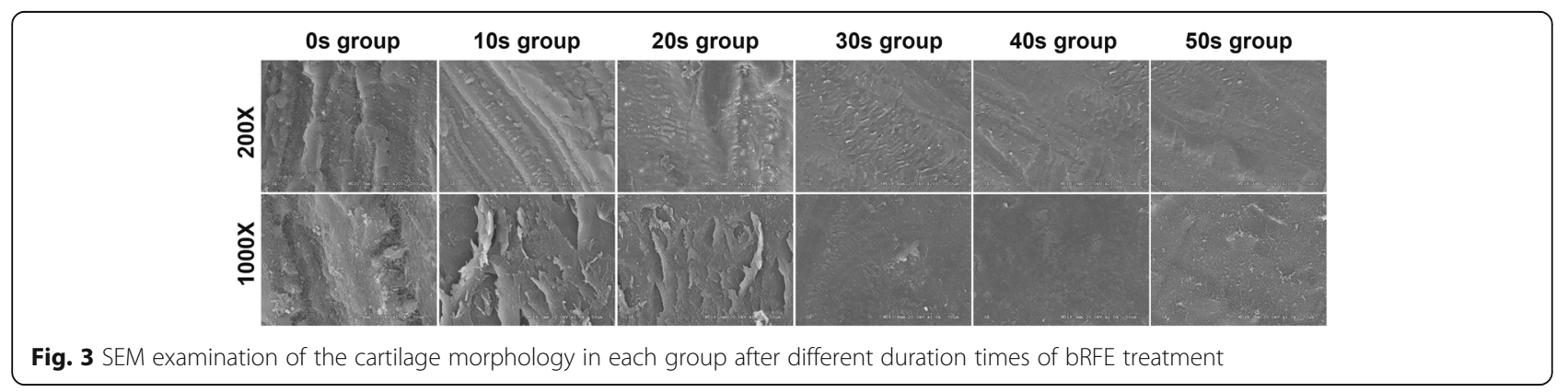




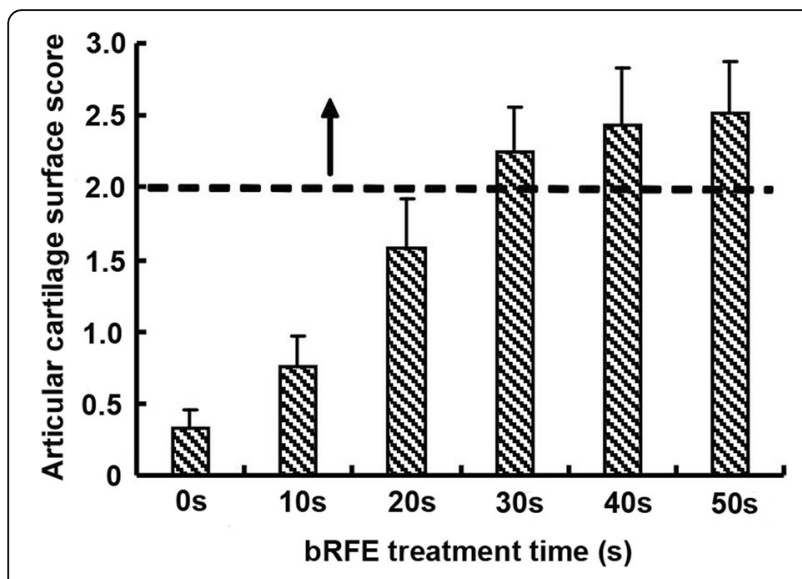

Fig. 4 Score of cartilage surface in each group after bRFE treatment at different duration times via SEM examination. The dotted line at surface score 2 is a standard for cartilage surface smoothing profiles [20]. No fluid flow was used in this study, as Edwards et al. [21] revealed a significant increase in temperature at $500 \mu \mathrm{m}$ underneath the cartilage surface but observed no change of temperature at the depth of $200 \mu \mathrm{m}$ and $2000 \mu \mathrm{m}$ when a $120-\mathrm{ml} / \mathrm{min}$ flow rate was applied in bRFE.

This study also presents with some limitations. Firstly, there were differences between the artificially induced chondral degeneration and the naturally and slowly occurring one. Secondly, the experiment was in vitro and unable to simulate ideal in vivo conditions. Thirdly, no fluid flow was applied while the actual arthroscopic procedure performed on patients requires fluid flow of varying speeds. Hence, caution is required when one tries to translate our conclusions into clinical settings, as the conclusions of a particular bRFE device come only from that device under the adopted treatment protocol. A different set

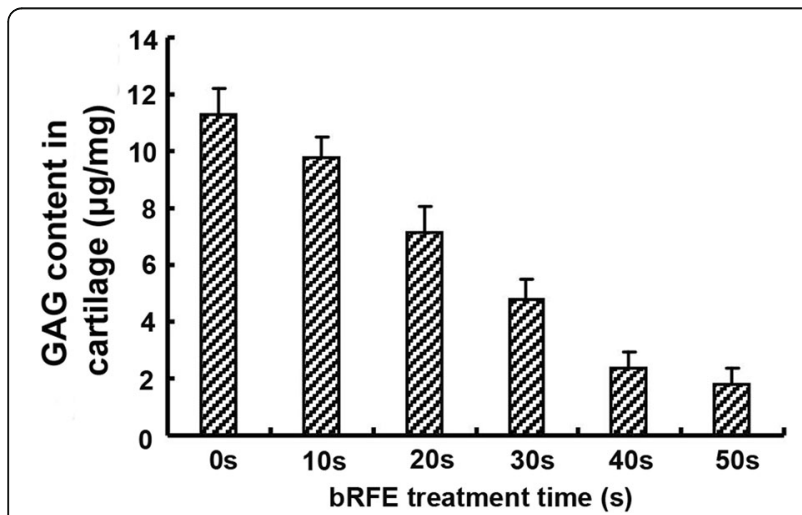

Fig. 5 GAG content in the cartilage in each group after bRFE treatment at different duration times of outcomes can be expected when any of the variables are changed (e.g., the probe is moved over the cartilage surface in a non-contact manner).

In conclusion, bRFE usage for chondroplasty could effectively trim and polish the cartilage lesion area. However, it induced a dose-dependent detrimental effect on chondrocytes and metabolic activity that negatively correlated with the treatment time. Therefore, cautions should be taken in the use of bRFE for treatment of articular cartilage injury.

\section{Abbreviations}

ANOVA: One-way analysis of variance; bRFE: Bipolar radiofrequency energy; DMB: Dimethylmethylene blue; GAG: Glycosaminoglycan; HE: Hematoxylin/ eosin; NBF: Neutral buffered formalin; OD: Optical density; SEM: Scanning electron microscopy

\section{Acknowledgements}

Not applicable.

\section{Authors' contributions}

LP performed the experiment and designed the study. YL performed the experiment and reviewed the manuscript. KZ performed the experiment. QC performed the experiment. LX performed data collection. YG performed statistical analysis. YH performed data collection. WZ performed statistical analysis. WL supervised the project. GZ performed language editing. ZD wrote the manuscript and designed the study. DW was responsible for the whole project and supervised the study. All authors read and approved the final manuscript.

\section{Funding}

This study was supported by the National Natural Science Foundation of China (81672234, 81902303, 81902558), Shenzhen Science and Technology Project (201606018, GJHZ20180416164801042, JCYJ20180305124912336), and Clinical Research Project of Shezhen Second People's Hospital

(20173357201814)

Availability of data and materials

All data generated or analyzed during this study are included in this published article.

\section{Competing interests}

The authors declare that they have no competing interests.

\section{Author details}

${ }^{1}$ Department of Sports Medicine, the First Affiliated Hospital of Shenzhen University, Shenzhen Second People's Hospital, Shenzhen 518035 ,

Guangdong, China. ${ }^{2}$ Shenzhen Key Laboratory of Tissue Engineering, the First Affiliated Hospital of Shenzhen University, Shenzhen Second People's Hospital, Shenzhen 518035, Guangdong, China. ${ }^{3}$ Department of Orthopaedics, Xiangya Hospital, Central South University, Changsha 410008, Hunan, China. ${ }^{4}$ Clinical Medical college of Shenzhen University, Shenzhen 518000, Guangdong, China. ${ }^{5}$ Guangzhou Medical University, Guangzhou 510182, Guangdong, China. ${ }^{6}$ Clinical College of Anhui Medical University, Affiliated Shenzhen Second Hospital, Shenzhen 518035, Guangdong, China. ${ }^{7}$ McGovern Medical School, University of Texas Health Science Center at Houston, Houston, TX, USA.

Received: 6 January 2020 Accepted: 5 March 2020

Published online: 12 March 2020

\section{References}

1. Deng ZH, Li YS, Gao X, Lei GH, Huard J. Bone morphogenetic proteins for articular cartilage regeneration. Osteoarthritis Cartilage. 2018;26:1153-61. https://doi.org/10.1016/j.joca.2018.03.007.

2. Kaab MJ, Bail HJ, Rotter A, Mainil-Varlet P, apGwynn I, Weiler A. Monopolar radiofrequency treatment of partial-thickness cartilage defects in the sheep 
knee joint leads to extended cartilage injury. Am J Sports Med. 2005;33: 1472-8. https://doi.org/10.1177/0363546505275013.

3. Gharaibeh M, Szomor A, Chen DB, MacDessi SJ. A retrospective study assessing safety and efficacy of bipolar radiofrequency ablation for knee chondral lesions. Cartilage. 2018;9:241-7. https://doi.org/10.1177/ 1947603517703731.

4. Edwards RB 3rd, Lu Y, Nho S, Cole BJ, Markel MD. Thermal chondroplasty of chondromalacic human cartilage. An ex vivo comparison of bipolar and monopolar radiofrequency devices. Am J Sports Med. 2002;30:90-7. https:// doi.org/10.1177/03635465020300012801.

5. Ryan A, Bertone AL, Kaeding CC, Backstrom KC, Weisbrode SE. The effects of radiofrequency energy treatment on chondrocytes and matrix of fibrillated articular cartilage. Am J Sports Med. 2003;31:386-91. https://doi.org/10.1177/ 036354650303.

6. Voloshin I, Morse KR, Allred CD, Bissell SA, Maloney MD, DeHaven KE. Arthroscopic evaluation of radiofrequency chondroplasty of the knee. Am J Sports Med. 2007;35:1702-7. https://doi.org/10.1177/0363546507304328.

7. Papalia R, Diaz Balzani L, Torre G, Paciotti M, Maffulli N, Denaro V. Radiofrequency energy in the arthroscopic treatment of knee chondral lesions: a systematic review. Br Med Bull. 2017;1. https://doi.org/10.1093/bmb/ldx016.

8. Spahn G, Hofmann GO, von Engelhardt LV. Mechanical debridement versus radiofrequency in knee chondroplasty with concomitant medial meniscectomy: 10-year results from a randomized controlled study. Knee Surg Sports Traumatol Arthrosc. 2016;24:1560-8. https:/doi.org/10.1007/s00167-015-3810-6.

9. Caffey S, McPherson E, Moore B, Hedman T, Vangsness CT Jr. Effects of radiofrequency energy on human articular cartilage: an analysis of 5 systems. Am J Sports Med. 2005;33:1035-9. https://doi.org/10.1177/ 0363546504271965.

10. Amiel D, Ball ST, Tasto JP. Chondrocyte viability and metabolic activity after treatment of bovine articular cartilage with bipolar radiofrequency: an in vitro study. Arthroscopy. 2004;20:503-10. https://doi.org/10.1016/j.arthro. 2004.03.018

11. Lu Y, Edwards RB 3rd, et al. Thermal chondroplasty with bipolar and monopolar radiofrequency energy: effect of treatment time on chondrocyte death and surface contouring. Arthroscopy. 2002;18:779-88. https://doi.org/ 10.1053/jars.2002.32840.

12. Hoemann CD, Sun J, Chrzanowski V, Buschmann MD. A multivalent assay to detect glycosaminoglycan, protein, collagen, RNA, and DNA content in milligram samples of cartilage or hydrogel-based repair cartilage. Anal Biochem. 2002;300:1-10. https://doi.org/10.1006/abio.2001.5436.

13. Lane JG, Amiel ME, Monosov AZ, Amiel D. Matrix assessment of the articular cartilage surface after chondroplasty with the holmium:YAG laser. Am J Sports Med. 1997;25:560-9. https://doi.org/10.1177/036354659702500421.

14. Goldberg-Bockhorn E, Schwarz S, Subedi R, et al. Laser surface modification of decellularized extracellular cartilage matrix for cartilage tissue engineering. Lasers Med. Sci. 2018;33:375-84. https://doi.org/10.1007/ s10103-017-2402-8.

15. Spahn G, Kahl E, Muckley T, Hofmann GO, Klinger HM. Arthroscopic knee chondroplasty using a bipolar radiofrequency-based device compared to mechanical shaver: results of a prospective, randomized, controlled study. Knee Surg Sports Traumatol Arthrosc. 2018;16:565-73. https://doi.org/10. 1007/s00167-008-0506-1.

16. Spahn G, Klinger HM, Muckley T, Hofmann GO. Four-year results from a randomized controlled study of knee chondroplasty with concomitant medial meniscectomy: mechanical debridement versus radiofrequency chondroplasty. Arthroscopy. 2010;26:S73-80. https://doi.org/10.1016/j. arthro.2010.02.030

17. Kaplan LD, Chu CR, Bradley JP, Fu FH, Studer RK. Recovery of chondrocyte metabolic activity after thermal exposure. Am J Sports Med. 2003;31:392-8. https://doi.org/10.1177/03635465030310031101.

18. Enochson L, Sonnergren HH, Mandalia VI, Lindahl A. Bipolar radiofrequency plasma ablation induces proliferation and alters cytokine expression in human articular cartilage chondrocytes. Arthroscopy. 2012;28:1275-82. https://doi.org/10.1016/j.arthro.2012.01.005.

19. Edwards RB 3rd, Hayashi K, Lu Y, Markel MD. The acute effects of radiofrequency energy in articular cartilage: an in vitro study. Arthroscopy. 2001;17:112-3. https://doi.org/10.1016/s0749-8063(01)70080-1.

20. Lu Y, Edwards RB 3rd, Cole BJ, Markel MD. Thermal chondroplasty with radiofrequency energy. An in vitro comparison of bipolar and monopolar radiofrequency devices. Am J Sports Med. 2001;29:42-9.
21. Edwards RB 3rd, Lu Y, Rodriguez E, Markel MD. Thermometric determination of cartilage matrix temperatures during thermal chondroplasty: comparison of bipolar and monopolar radiofrequency devices. Arthroscopy. 2002;18: 339-46. https://doi.org/10.1053/jars.2002.29893.

\section{Publisher's Note}

Springer Nature remains neutral with regard to jurisdictional claims in published maps and institutional affiliations.
Ready to submit your research? Choose BMC and benefit from:

- fast, convenient online submission

- thorough peer review by experienced researchers in your field

- rapid publication on acceptance

- support for research data, including large and complex data types

- gold Open Access which fosters wider collaboration and increased citations

- maximum visibility for your research: over $100 \mathrm{M}$ website views per year

At BMC, research is always in progress.

Learn more biomedcentral.com/submissions 